Research Paper

\title{
The prognostic value of age in non-metastatic gastric cancer after gastrectomy: a retrospective study in the U.S. and China
}

Jieyun Zhang1,2*, Lu Gan ${ }^{3 *}$, Mi-die Xu2 $4^{*}$, Mingzhu Huang1, 2, Xiaowei Zhang1, 2, Yiwei Gong1, 2, Xi Wang Guanzhen $\mathrm{Yu}^{6}$, Weijian Guo ${ }^{1,2}$

1. Department of Medical Oncology, Fudan University Shanghai Cancer Center, Shanghai 200032, P.R. China

2. Department of Oncology, Shanghai Medical College, Fudan University, Shanghai 200032, P.R. China

3. Department of Medical Oncology, Zhongshan Hospital, Fudan University, Shanghai 200032, China

4. Department of Pathology, Fudan University Shanghai Cancer Center, Shanghai 200032, P.R. China

5. Department of Oncology, the 117th Hospital of PLA, 14 Lingyin Road, Hangzhou 310013, P.R. China

6. Department of Oncology, East Hospital, Tongji University School of Medicine, Shanghai 200120, P.R. China

* These authors contributed equally to this work.

$\triangle$ Corresponding author: Weijian Guo, Department of Medical Oncology, Fudan University Shanghai Cancer Center, Shanghai 200032, P.R. China; E-mail: guoweijian1@sohu.com

(c) Ivyspring International Publisher. This is an open access article distributed under the terms of the Creative Commons Attribution (CC BY-NC) license (https://creativecommons.org/licenses/by-nc/4.0/). See http://ivyspring.com/terms for full terms and conditions.

Received: 2017.07.26; Accepted: 2017.11.29; Published: 2018.03.08

\begin{abstract}
Purpose: We explored the influence of age on clinicopathologic features and survival of patients with M0 gastric cancer (GC).

Methods: 16856 GC patients from Surveillance, Epidemiology and End Results (SEER) database and 1037 GC patients from Chinese multiple centers were enrolled in the U.S. and Chinese cohort, respectively. 50-year-old was treated as cutoff age. Propensity score method was used to carry out a 1:1 paired match.

Results: In the U.S. cohort, we found that younger patients presented poor tumor behavior. However, in spite of worse outcome in stage I IV cohort, young group showed better 3-year survival in M0 patients, especially for those who underwent a total gastrectomy. In a matched analysis, a better prognosis was still observed in younger group. The prognostic value of age was also validated in MO GC patients with gastrectomy in Chinese cohort.

Conclusions: In spite of the worse outcome in survival curve of stage I IV GC cohort, young patients with gastrectomy presented favorable survival in $M 0$ subgroup. It is also applicable in China. Early diagnosis and treatment should be taken seriously in young GC patients since they often possess poorer characteristics but benefited more from gastrectomy.
\end{abstract}

Key words: Gastric cancer, age, SEER, survival analysis, retrospective study

\section{Introduction}

Gastric cancer (GC) is one of the leading causes of cancer-related death around the world [1, 2]. While old age is known as a significant risk factor for GC, more than half of GC patients are over 60 years old and the median age at diagnosis is 70 years [3]. Decades ago, GC was the most common cancer throughout the world [3]. Recently, the overall incidence of GC has steadily declined due to an outcome of advances in the management of gastrointestinal carcinoma. However, the incidence rate of GC in the population younger than 50 years has inversely increased [4]. This could be explained by the fact that GC routinely screening in population aged 50 and younger has not been taken seriously in clinical practice [5]. 
It's been reported that younger patients tend to have following typical characteristics. Helicobacter pylori infection, family history, diffuse type, and poorly differentiated tumor were more frequent in the young patients rather than the elderly patients [6-10]. Meanwhile, a controversial issue is whether age is a prognostic factor of gastric cancer. Many studies have reported that young patients with GC had a worse prognosis than their elderly counterparts [7, 11]. However, other reports presented that the survival of young GC patients was as good as or even better than elderly patients [12-15]. These diverse findings between their small-sample studies could be explained by the neglect of screening and early detection of GC in the younger population. On the other hand, it has been found that young patients also have a better tolerance of surgeries and a stronger will of treatments [16]. Moreover, as the elderly population remains predominant amongst GC patients, most studies compared a smaller number of young patients with a much larger number of the elderly. This gave rise to a bias between two unbalanced groups [14]. We suggested that the tendency to metastasis in younger GC patients contributed to a poorer outcome. To verify our hypothesis, we observed the cancer-specific survival (CSS) in different age groups of GC patients and focused on the age-stratified survival of non-metastatic (M0) GC. To explore if younger patients had unique clinicopathological and outcome, we used the data from Surveillance, Epidemiology, and End Results (SEER) database to analysis the effect of age on M0 GC.

As we know, the highest incidence rate of GC occurs in Eastern Asia while the lowest is in Northern America [17]. In the meantime, the prognosis for gastric cancer is better for patients in the U.S. than in Asia [18]. Considering the various incidence and mortality rates, the results of analysis based on population in U.S. aren't applicable in Asia without validation. For this reason, we assessed clinicopathological data from a multicentric Chinese cohort, and performed survival analysis to explore whether the findings of this study can be applied in China.

\section{Materials and Methods}

\section{Patient selection in the U.S. cohort}

Surveillance, Epidemiology, and End Results (SEER) database released in April 2017 was used as our data source. The SEER database is publicly available for studies of epidemiology and health policy. It covers almost $30 \%$ population in the US and consists data from 18 population-based registries from 1973 to 2012. The SEER Program registries routinely collect data on patient demographics, primary tumor site, tumor morphology and stage at diagnosis, first course of treatment, and follow-up for vital status. The data of cancer incidence, mortality and trends in the United States reported by SEER are provided and updated annually by the Centers for Disease Control and Prevention, the American Cancer Society, the National Cancer Institute, and the North American Association of Central Cancer Registries.

Using the population-based database, we identified patients with primary GC (International Classification of Diseases for Oncology, Third Edition [ICD-O-3], codes C16.1-16.6, C16.8-16.9) who were 18 or older at diagnosis between 1 January 2004 and 31 December 2012. Patients were included with histological types as adenocarcinoma and signet ring cell carcinoma. Patients were excluded if they had diagnostic confirmation by autopsy or death certificate, unknown TNM stage or cause of death or marital status or survival months, or tumors located at the cardia or esophagogastric junction site (ICD-O-3, code C16.0). After applying these inclusion criteria, 16856 patients were enrolled in our analytic cohort.

\section{Variables in the U.S. cohort}

Outcomes of interest in SEER database were overall, cancer-specific and other-cause mortalities. Cancer-specific survival (CSS) was calculated from the date of GC diagnosis to the date of death attributed to GC. Deaths attributed to GC were treated as events. Patients who died from other causes or were still alive at the time of the last follow-up were treated as censored observations and 31 December 2012 was taken as the follow-up cutoff date of our study.

We extracted following information of patients from the SEER database: age at diagnosis, sex, race, marital status, histological type, primary site, TNM stage, depth of invasion, lymph node involvement, cause of death, histological grade, surgery, and radiotherapy. Age 50 at diagnosis was treated as a cut-off age because population aged 50 and younger lacked routinely screening and was reported to have high-frequency microsatellite instability $[19,20]$. Race was classified as white, black, American Indian or Alaska Native, Asian or Pacific Islander, and others. Marital status at diagnosis was categorized into married, single, divorced or separated, and widowed groups. Data of histological types, histology grade and primary site were coded according to ICD-O-3. Data of stage, depth of invasion, and lymph node involvement were all restaged according to the 7th edition of AJCC Cancer Staging Manual [21]. 


\section{Patient selection in Chinese cohort}

A total of 1037 patients who were diagnosed with gastric cancer underwent gastrectomy in Fudan University Shanghai Cancer Center, Shanghai, China, and Changhai Hospital, Second Military Medical University, Shanghai, China, from 2000 to 2012. None of these patients had synchronous benign disease or cancers, previous gastrointestinal diseases, abdominal surgery, chemotherapy or radiotherapy. Patients without sufficient clinicopathological information, or patients suffered from more than on primary tumors or remnant gastric cancer, or those died within two months of surgery, were all excluded. Our study was approved by the Fudan University Shanghai Cancer Center ethics committee and Institutional Review Board of Second Military Medical University.

\section{Variables in the Chinese cohort}

Overall survival (OS) was defined from the date of diagnosis to the date of death. Patients were followed up until death of any cause or our study end, except for those lost to follow-up. Information gathered for China patients included age, sex, Lauren classification, depth of invasion, lymph node involvement, primary site and grade. After collecting data, these variables were coded and categorized. Patients younger than 50 were classified into young group and the others were in old group. Grade I and II were grouped as well/moderately differentiated, while grade III and IV as poorly differentiated/undifferentiated.

\section{Statistical Analysis}

Baseline characteristics of patients and tumors were compared across age groups by Pearson chi-square test (categorical data), and Wilcoxon-Mann-Whitney test (ranked data). The CSS rate was calculated by Kaplan-Meier curve, and differences between curves was clarified by Log-rank (Mantel-Cox) test. Multivariate Cox proportional hazard models were applied to determine factors related to survival outcomes with adjustment for possible confounders, and HRs with 95\% CIs were calculated. We presented a forest plot to summarize the hazard ratios of elderly versus young group in subgroups by univariate Cox regression analysis, as well as demonstrate each prognostic factors effect on survival.

Considering diverse factors across age groups, we carried out a matched case-control analysis. Psmatch2 was used to match each young patient to one elderly patient, according to race, sex, histological grade, TNM stage, and histological types. As an extension packages in Stata, psmatch2 is designed for the propensity score matching method. We used Stata statistical software, version 12.0 (StataCorp, College Station, TX) to perform all the statistical analysis. Two-sided $\mathrm{P}$ values $<0.05$ were considered statistical significance.

\section{Results}

\section{Baseline Characteristic of patients}

According to the inclusion criteria, we finally enrolled 16,856 eligible GC patients from SEER in our study. Sex, race, marital status, histological type, primary site, TNM stage, depth of invasion, lymph node involvement, histological grade, surgery, and radiotherapy showed differences between the young (18 - 49 years old) and the elderly group ( $\geq 50$ years old). The detailed information was shown in Table S1. Among them, 12,216 patients at M0 stage were enrolled in our following study. 1338 (10.95\%) patients were in the young group and 10,878 (89.05\%) were in the elderly group. The median follow-up time to check up on patients was $21(9-48)$ months and 16 (5 - 41) months in the young and the elderly group, respectively. There were significant differences in sex, race, marital status, histological type, primary site, TNM stage, depth of invasion, lymph node involvement, histological grade, surgery and radiotherapy between the young and the elderly groups. The younger patients presented with a higher proportion of differing characteristics such as female, black, married, single, lymph node involvement, deeper invasion, diffuse carcinoma and signet ring cell carcinoma, stage II \& III, and grade III \& IV, than the elderly patients. Total or total gastrectomies and radiotherapies were more frequently performed on the younger patients. Patient demographics, pathological characteristics of tumors and treatment types based on age are summarized in Table 1.

Additionally, 1037 multicentric Chinese M0 GC patients diagnosed from 2000 to 2012 were included in our Chinese cohort and $23.53 \%$ of them were younger than 50 years. Female, shallower invasion, lower site and poorer differentiation were observed in young patients, which were all presented in Table 2.

\section{Effect of Age on Cancer-specific Survival in the U.S. cohort}

The unadjusted CSS by age in stage I IV GC patients in the U.S. cohort was calculated by Kaplan-Meier curves. The 3-year CSS in the young and the elderly patients was $38.36 \%$ and $42.18 \%$, respectively. It was shown that elderly patients had a significantly better prognosis in the univariate log-rank test $(\mathrm{P}<0.001)$. (Figure $1 \mathrm{~A})$ 
Table 1. Characteristics of patients with non-metastatic gastric cancer by age, young versus elderly. SEER $2004-2012(n=12216)^{a}$

\begin{tabular}{|c|c|c|c|c|}
\hline \multirow[t]{2}{*}{ Characteristics } & \multirow{2}{*}{$\frac{\text { Total }}{12216(100)}$} & \multirow{2}{*}{$\begin{array}{l}<50 \\
1338(10.95)\end{array}$} & \multirow{2}{*}{$\begin{array}{l}\geq 50 \\
10878(89.05)\end{array}$} & \multirow[t]{2}{*}{ Pvalue ${ }^{b}$} \\
\hline & & & & \\
\hline \multicolumn{5}{|l|}{ Median Follow-up } \\
\hline Months (IQR) & $16(6-42)$ & $21(9-48)$ & $16(5-41)$ & \\
\hline Age (IQR) & $70(59-79)$ & $44(39-47)$ & $73(64-80)$ & \\
\hline Sex & & & & 0.01 \\
\hline Male & $6793(55.61)$ & $700(52.32)$ & $6093(56.01)$ & \\
\hline Female & $5423(44.39)$ & $638(47.68)$ & 4785 (43.99) & \\
\hline Race & & & & 0.001 \\
\hline White & $7254(59.38)$ & $762(56.95)$ & $6492(59.68)$ & \\
\hline Black & $2014(16.49)$ & $272(20.33)$ & $1742(16.01)$ & \\
\hline American Indian/Alaska Native & $115(0.94)$ & $17(1.27)$ & $98(0.9)$ & \\
\hline Asian or Pacific Islander & $2792(22.86)$ & $283(21.15)$ & $2509(23.06)$ & \\
\hline Unknown & $41(0.34)$ & $4(0.3)$ & $37(0.34)$ & \\
\hline Marital status & & & & $<0.001$ \\
\hline Married & $7094(58.07)$ & $863(64.5)$ & $6231(57.28)$ & \\
\hline Single (never married) & $1658(13.57)$ & $350(26.16)$ & 1308 (12.02) & \\
\hline Divorced/Separated & $1079(8.83)$ & $116(8.67)$ & $963(8.85)$ & \\
\hline Widowed & $2385(19.52)$ & $9(0.67)$ & $2376(21.84)$ & \\
\hline Histological type & & & & $<0.001$ \\
\hline Adenocarcinoma, NOS & $5852(47.9)$ & $443(33.11)$ & 5409 (49.72) & \\
\hline Adenocarcinoma, intestinal type & $2042(16.72)$ & $84(6.28)$ & $1958(18)$ & \\
\hline Carcinoma, diffuse type & $803(6.57)$ & $145(10.84)$ & $658(6.05)$ & \\
\hline Tubular adenocarcinoma & $148(1.21)$ & $6(0.45)$ & $142(1.31)$ & \\
\hline Papillary adenocarcinoma, NOS & $39(0.32)$ & $5(0.37)$ & $34(0.31)$ & \\
\hline Mucinous adenocarcinoma & $233(1.91)$ & $24(1.79)$ & 209 (1.92) & \\
\hline Signet ring cell carcinoma & 3099 (25.37) & $631(47.16)$ & $2468(22.69)$ & \\
\hline Site & & & & 0.046 \\
\hline Fundus of stomach & $573(4.69)$ & $52(3.89)$ & $521(4.79)$ & \\
\hline Body of stomach & $1493(12.22)$ & $150(11.21)$ & $1343(12.35)$ & \\
\hline Gastric antrum & $4235(34.67)$ & $442(33.03)$ & $3793(34.87)$ & \\
\hline Pylorus & $678(5.55)$ & $73(5.46)$ & $605(5.56)$ & \\
\hline Lesser curvature of stomach, NOS & $1768(14.47)$ & $189(14.13)$ & 1579 (14.52) & \\
\hline Greater curvature of stomach, NOS & $722(5.91)$ & $86(6.43)$ & $636(5.85)$ & \\
\hline Overlapping lesion of stomach & $1178(9.64)$ & $158(11.81)$ & $1020(9.38)$ & \\
\hline Stomach, NOS & $1569(12.84)$ & $188(14.05)$ & $1381(12.7)$ & \\
\hline Stage & & & & $<0.001$ \\
\hline I & $4453(36.45)$ & $342(25.56)$ & $4111(37.79)$ & \\
\hline II & $3708(30.35)$ & $432(32.29)$ & $3276(30.12)$ & \\
\hline III & $4055(33.19)$ & $564(42.15)$ & 3491 (32.09) & \\
\hline Depth of invasion & & & & $<0.001$ \\
\hline $\mathrm{T} 1$ & $3765(30.82)$ & $287(21.45)$ & 3478 (31.97) & \\
\hline $\mathrm{T} 2$ & $1446(11.84)$ & $143(10.69)$ & 1303 (11.98) & \\
\hline T3 & $5736(46.95)$ & $733(54.78)$ & 5003 (45.99) & \\
\hline $\mathrm{T} 4$ & $1269(10.39)$ & $175(13.08)$ & $1094(10.06)$ & \\
\hline Lymph node involvement & & & & $<0.001$ \\
\hline N0 & $6071(49.7)$ & $528(39.46)$ & $5543(50.96)$ & \\
\hline N1 & $1670(13.67)$ & $193(14.42)$ & 1477 (13.58) & \\
\hline N2 & $2250(18.42)$ & $295(22.05)$ & 1955 (17.97) & \\
\hline N3 & $2225(18.21)$ & $322(24.07)$ & 1903 (17.49) & \\
\hline Grade & & & & $<0.001$ \\
\hline Grade I & $534(4)$ & $25(1.87)$ & $509(4.68)$ & \\
\hline Grade II & $2940(24.07)$ & $129(9.64)$ & $2811(25.84)$ & \\
\hline Grade III & $7538(61.71)$ & $1038(77.58)$ & $6500(59.75)$ & \\
\hline Grade IV & $253(2.07)$ & $38(2.84)$ & $215(1.98)$ & \\
\hline Cell type not determined & $951(7.78)$ & $108(8.07)$ & $843(7.75)$ & \\
\hline Surgery & & & & $<0.001$ \\
\hline No surgery & $2332(19.09)$ & $204(15.25)$ & $2128(19.56)$ & \\
\hline Non-Total or Non-near-total gastrectomy & $7780(63.69)$ & 799 (59.72) & $6981(64.18)$ & \\
\hline Total or near total gastrectomy & $2104(17.22)$ & $335(25.04)$ & $1769(16.26)$ & \\
\hline Radiotherapy & & & & $<0.001$ \\
\hline No radiotherapy & $8692(71)$ & $750(56.05)$ & $7942(73)$ & \\
\hline Radiotherapy & $3322(27.19)$ & $563(42.08)$ & $2759(25.36)$ & \\
\hline Radiotherapy unknown & $202(1.65)$ & $25(1.87)$ & $177(1.63)$ & \\
\hline
\end{tabular}

Abbreviation: NOS= no other specific; SEER=Surveillance, Epidemiology and End Results; IQR=interquartile range

aData are presented as No. (Percentage) of patients.

$\mathrm{bP}$ value of the Chi-square test or Wilcoxon-Mann-Whitney test comparing young and elderly groups

cBeing restaged according to the criteria of AJCC Cancer Staging Manual (7th edition, 2010) 
Table 2. Characteristics of patients with non-metastatic gastric cancer by age, young versus elderly. Chinese cohort 2000-2012 $(n=1037)$

\begin{tabular}{|c|c|c|c|c|}
\hline \multirow[t]{2}{*}{ Characteristics } & Total & $<50$ & $\geq 50$ & \multirow[t]{2}{*}{ P value } \\
\hline & $1037(100)$ & $244(23.53)$ & $793(76.47)$ & \\
\hline \multicolumn{5}{|l|}{ Median Follow-up } \\
\hline Months (IQR) & $45(18-73)$ & $58.5(26-76)$ & $42(16-72)$ & \\
\hline Age (IQR) & $60(50-69)$ & $43(38-47)$ & $64(57-71)$ & \\
\hline Sex & & & & $<0.001$ \\
\hline Male & $724(69.82)$ & $148(60.66)$ & $576(72.64)$ & \\
\hline Female & $313(30.18)$ & $96(39.34)$ & $217(27.36)$ & \\
\hline Lauren classification & & & & 0.001 \\
\hline Intestinal & $773(74.54)$ & $166(68.03)$ & $607(76.54)$ & \\
\hline Mixed & $39(3.76)$ & $5(2.05)$ & $34(4.29)$ & \\
\hline Diffuse & $225(21.7)$ & $73(29.92)$ & $152(19.17)$ & \\
\hline Depth of invasion & & & & 0.002 \\
\hline $\mathrm{T} 1$ & $185(17.84)$ & $62(25.41)$ & $123(15.51)$ & \\
\hline $\mathrm{T} 2$ & $168(16.2)$ & $43(17.62)$ & 125 (15.76) & \\
\hline T3 & $515(49.66)$ & $101(41.39)$ & $414(52.21)$ & \\
\hline $\mathrm{T} 4$ & $169(16.3)$ & $38(15.57)$ & $131(16.52)$ & \\
\hline Lymph node involvement & & & & 0.905 \\
\hline N0 & $384(37.03)$ & $89(36.48)$ & $295(37.2)$ & \\
\hline N1 & $176(16.97)$ & $45(18.44)$ & $131(16.52)$ & \\
\hline N2 & $196(18.9)$ & $44(18.03)$ & $152(19.17)$ & \\
\hline N3 & $281(27.1)$ & $66(27.05)$ & $215(27.11)$ & \\
\hline Site & & & & 0.001 \\
\hline Upper & $184(17.74)$ & $24(9.84)$ & $160(20.18)$ & \\
\hline Middle & $330(31.82)$ & $88(36.07)$ & $242(30.52)$ & \\
\hline Lower & $474(45.71)$ & $124(50.82)$ & $350(44.14)$ & \\
\hline Diffuse & $49(4.73)$ & $8(3.28)$ & $41(5.17)$ & \\
\hline Grade & & & & $<0.001$ \\
\hline Undifferentiated/poor & $644(62.1)$ & $191(78.28)$ & $453(57.12)$ & \\
\hline Well/moderate & $323(31.15)$ & $42(17.21)$ & $281(35.44)$ & \\
\hline Unknown & $70(6.75)$ & $11(4.51)$ & $59(7.44)$ & \\
\hline Outcome & & & & $<0.001$ \\
\hline Alive & $577(55.64)$ & $167(68.44)$ & $410(51.7)$ & \\
\hline Dead & $460(44.36)$ & 77 (31.56) & $383(48.3)$ & \\
\hline
\end{tabular}

Then, we used Kaplan-Meier curve to determine the CSS of patients with M0 GC in Figure 1B. The curves showed that the CSS was significantly different according to the univariate log-rank test $(\mathrm{P}=0.005)$, and that the young patients had improved their CSS rather than the elderly (Table 3 ). The 3-year CSS rate was $56.6 \%$ of patients in the young group and $52.77 \%$ of patients in the elderly group. Then, we performed Cox regression to adjust all significant factors in multivariate analysis and confirmed that some of them were still independent prognostic factors for CSS, including age, primary site, race, marital status, grade, histological type, TNM stage, surgery and radiotherapy.

\section{Subgroup Analyses}

Further univariate subgroup analyses for M0 GC were shown in Figure 2. A forest plot of hazard ratio (HR) was made to present an exploratory subgroup analysis for CSS. In some subgroups with wide confidence intervals (CIs) covering 1.00, HR had no statistical significance and age failed to be a risk factor. These subgroups could be identified as considerable confounders for effect of age in survival of patients with M0 GC. Among them, surgery was chosen as our candidate subgroup for further exploration.

\section{Stratified Analysis of Age on Cancer-specific Survival According to Surgery}

In order to make sure whether the survival advantage of young patients in M0 GC still existed after different surgical selection, we then carried out further exploration on the different prognosis between the young and the elderly patients in each subgroup of surgery. We made an analysis of the differences of age on 3-year CSS according to surgery. Kaplan-Meier curves are shown in Figure 1C, Figure $1 \mathrm{D}$, and Figure 1E. For patients who received total or near-total gastrectomy, it was shown in Table S2 that the younger patients have a better CSS than the elderly patients by the univariate log-rank analysis $(\mathrm{P}=0.021)$. However, age was not significantly more associated with CSS for patients who received no surgery or non-total or non-near-total gastrectomy. After adjusting the possible confounding factors in the multivariate Cox regression analysis, advanced age was defined as an independent risk factor for CSS in the subgroup of M0 GC patients who received total or near-total gastrectomy (Ref: $<50 ; \geq 50$ : $H R=1.34,95 \%$ $\mathrm{CI}=1.11-1.62, \mathrm{P}=0.003)$.

\section{Survival Analysis in Matched Group}

In our study, the numbers of young patients were much less than the elderly patients (1338 vs 10878). In order to rule out the interference of difference in baseline characteristics between the two groups, we performed a 1:1 matched case-control analysis and matched each young patient to one elderly patient. By this propensity score matching method, we enrolled 2676 patients and 1338 for each age group. The median follow-up time for patients were 21 (9-48) and 20 (7-74) months respectively. There was no statistically significant difference in sex $(\mathrm{P}=0.938)$, race $(\mathrm{P}=0.965)$, histological type $(\mathrm{P}=0.926)$, primary site $(\mathrm{P}=0.949)$, TNM stage $(\mathrm{P}=0.999)$, and histological grade $(\mathrm{P}=0.943)$ between the two groups. The detailed demographics, pathological characteristics of tumors and treatments received are shown in Table 4. Furthermore, the younger group $(56.6 \%)$ demonstrated a better 3-year CSS than the elderly $(48.76 \%$ ) (Figure 3 ) in both the univariate Log-rank test $(\mathrm{P}<0.001)$ and the multivariate Cox regression analysis (Ref: $<50 ; \geq 50$ : $H R=1.39,95 \%$ $\mathrm{CI}=1.23-1.57, \quad \mathrm{P}<0.001$ ) (Table S3). This outcome proves that our original analysis was credible. 
A

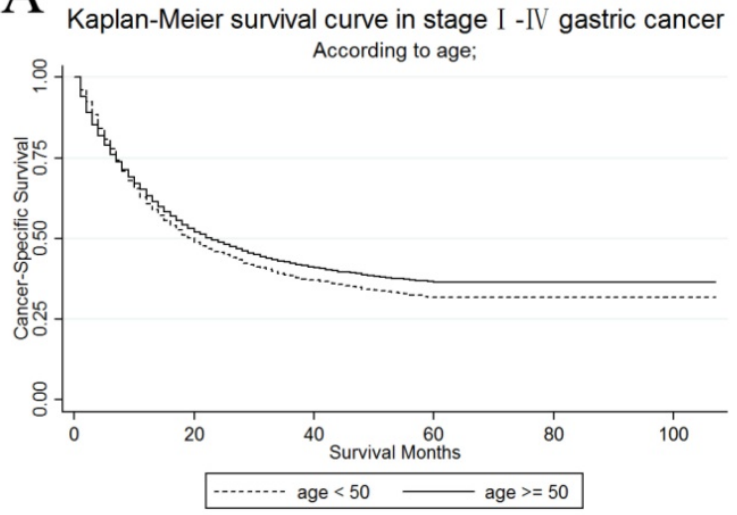

C

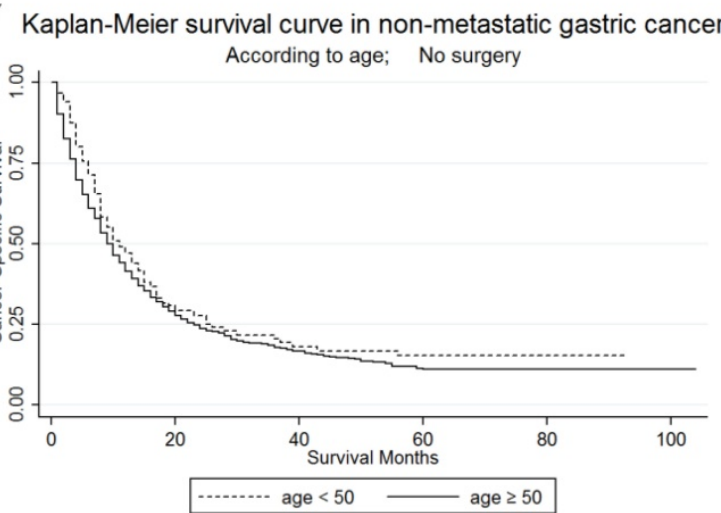

$\mathrm{E}$

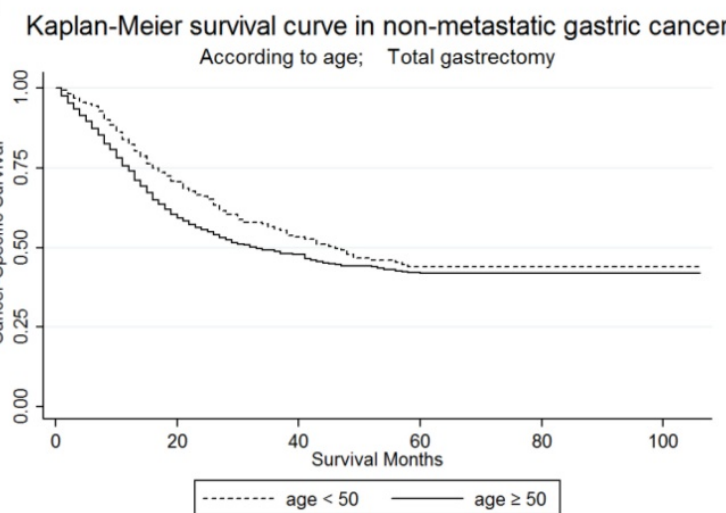

B

Kaplan-Meier survival curve in non-metastatic gastric cancer

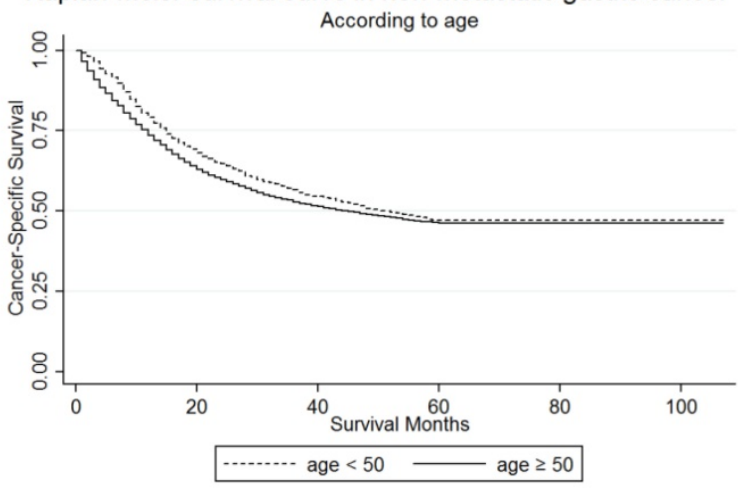

$\mathrm{D}$

Kaplan-Meier survival curve in non-metastatic gastric cancer

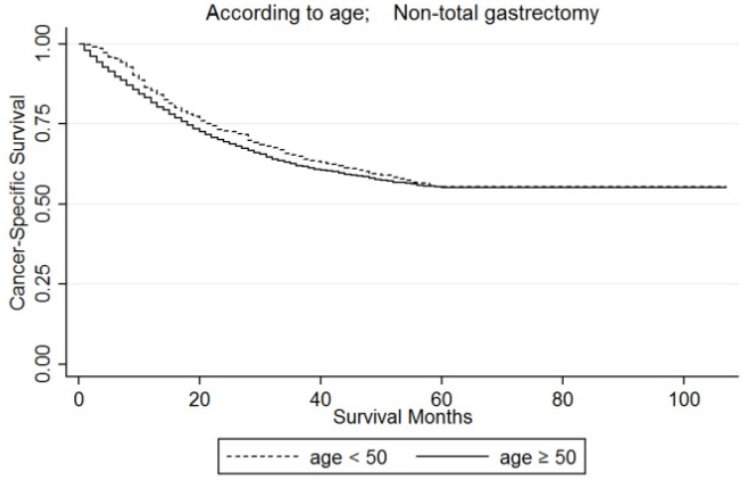

$\mathrm{F}$

Chinese cohort: Kaplan-Meier survival curve in non-metastatic gastric cancer According to age

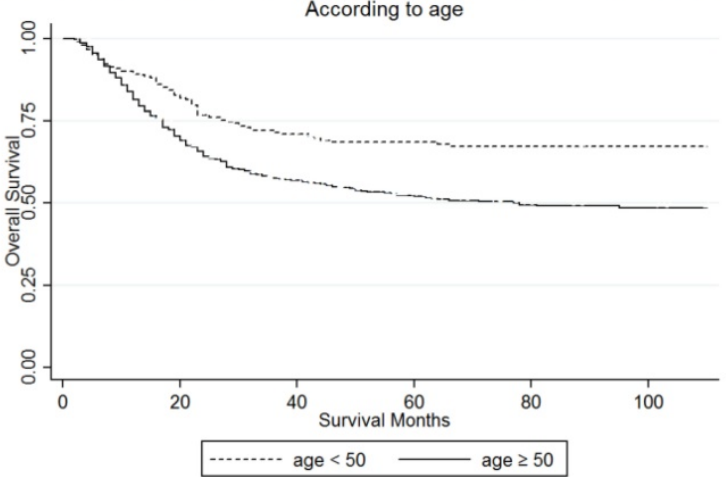

Figure 1: Kaplan-Meier survival curves according to age, young versus elderly patients (Figure 1A-1E show survival in the U.S. cohort while Figure IF in Chinese cohort). (A) The cancer-specific survival of stage I IV gastric cancer: $X^{2}=6.21, P=0.0127$; (B) The cancer-specific survival of M0 gastric cancer: $X^{2}=7.89, P=0.005(C)$ The cancer-specific survival of $M 0$ gastric cancer without surgery: $X^{2}=3.1, P=0.0781$; $(D)$ The cancer-specific survival of $M 0$ gastric cancer with non-total gastrectomy: $X^{2}=2.12, P=0.1457(E)$ The cancer-specific survival of $M 0$ gastric cancer with total gastrectomy: $X^{2}=5.33, P=0.021 ;(F)$ The overall survival of M0 GC patients with gastrectomy (Chinese cohort): $X^{2}=19.33, P<0.001$

\section{Survival analysis in Chinese cohort}

To confirm that younger age is still a protective factor of survival for M0 GC patients with surgery in China, we further make survival analysis in Chinese cohort. The Kaplan-Meier curves by age were shown in Figure 1F. Young patients had a significantly better overall survival than old patients $(71.44 \%$ versus
$52.90 \%)$ in the log-rank test $(\mathrm{P}<0.001)$. After adjusting for age, Lauren classification, depth of invasion, lymph node involvement, site and grade, age was still an independent prognostic factor while old patients had poorer outcome than young patients (Ref: <50; 250: $\mathrm{HR}=1.79,95 \% \mathrm{CI}=1.39-2.307, \mathrm{P}<0.001)$, which accorded with the result in the U.S. cohort (Table 5). 


\section{Discussion}

The occurrence of GC is generally regarded to be relevant to the age of the patient and it usually occurs in people aged over 50 years old. Previous researches reported that the proportion of young patients with GC ranged from $2 \%$ to $15.1 \%$, according to different cutoff-age criterion [3, 12, 22-24]. In this study, we treated 50 years as the cutoff age and those younger would be classified as the young group. This study has also showed that the proportion of young age in M0 GC was $10.95 \%$ in the U.S. cohort and $23.53 \%$ in Chinese cohort.

Table 3. Univariate and multivariate survival analysis for gastric cancer-specific survival (CSS) predictors. SEER 2004-2012 ( $n=12216$ )

\begin{tabular}{|c|c|c|c|c|c|c|}
\hline \multirow[t]{2}{*}{ Variable } & \multirow[b]{2}{*}{ 3-year CSS } & \multicolumn{2}{|c|}{ Univariate Analysis } & \multicolumn{2}{|c|}{ Multivariate Analysis } & \multirow[b]{2}{*}{ P Value } \\
\hline & & Log rank $\chi^{2}$ & P Value & HR & $95 \% \mathrm{CI}$ & \\
\hline Age & & 7.89 & 0.005 & & & \\
\hline$<50$ & $56.60 \%$ & & & Reference & & \\
\hline$\geq 50$ & $52.77 \%$ & & & 1.32 & $1.20-1.45$ & $<0.001$ \\
\hline Primary Site & & 235.07 & $<0.001$ & & & \\
\hline Fundus of stomach & $44.60 \%$ & & & Reference & & \\
\hline Body of stomach & $54.74 \%$ & & & 0.92 & $0.79-1.07$ & 0.264 \\
\hline Gastric antrum & $57.12 \%$ & & & 0.9 & $0.79-1.03$ & 0.132 \\
\hline Pylorus & $51.35 \%$ & & & 1.04 & $0.88-1.24$ & 0.625 \\
\hline Lesser curvature of stomach, NOS & $61.48 \%$ & & & 0.76 & $0.65-0.88$ & $<0.001$ \\
\hline Greater curvature of stomach, NOS & $56.10 \%$ & & & 1.01 & 0.85-1.19 & 0.943 \\
\hline Overlapping lesion of stomach & $39.67 \%$ & & & 1.01 & $0.87-1.17$ & 0.879 \\
\hline Stomach, NOS & $43.55 \%$ & & & 1.08 & $0.93-1.24$ & 0.302 \\
\hline Sex & & 5.45 & 0.0195 & & & \\
\hline Male & $54.01 \%$ & & & Reference & & \\
\hline Female & $52.19 \%$ & & & 0.97 & $0.91-1.03$ & 0.283 \\
\hline Race & & 152.62 & $<0.001$ & & & \\
\hline White & $50.28 \%$ & & & Reference & & \\
\hline Black & $49.28 \%$ & & & 1.03 & $0.95-1.11$ & 0.517 \\
\hline American Indian/Alaska Native & $46.62 \%$ & & & 1.06 & $0.81-1.39$ & 0.685 \\
\hline Asian or Pacific Islander & $63.25 \%$ & & & 0.76 & $0.71-0.82$ & $<0.001$ \\
\hline Unknown & $83.28 \%$ & & & 0.38 & $0.17-0.84$ & 0.017 \\
\hline Marital status & & 107.47 & $<0.001$ & & & \\
\hline Married & $56.30 \%$ & & & Reference & & \\
\hline Single (never married) & $50.86 \%$ & & & 1.07 & $0.98-1.17$ & 0.118 \\
\hline Divorced/Separated & $52.42 \%$ & & & 1.07 & $0.96-1.18$ & 0.229 \\
\hline Widowed & $45.41 \%$ & & & 1.33 & $1.23-1.44$ & $<0.001$ \\
\hline Grade & & 268.73 & $<0.001$ & & & \\
\hline Grade I & $79.71 \%$ & & & Reference & & \\
\hline Grade II & $62.46 \%$ & & & 1.46 & $1.19-1.80$ & $<0.001$ \\
\hline Grade III & $48.81 \%$ & & & 1.86 & $1.52-2.28$ & $<0.001$ \\
\hline Grade IV & $42.75 \%$ & & & 2.31 & $1.77-3.02$ & $<0.001$ \\
\hline Cell type not determined & $47.57 \%$ & & & 1.44 & $1.15-1.80$ & 0.001 \\
\hline Histological type & & 167.02 & $<0.001$ & & & \\
\hline Adenocarcinoma, NOS & $52.88 \%$ & & & Reference & & \\
\hline Adenocarcinoma, intestinal type & $65.74 \%$ & & & 0.86 & $0.78-0.94$ & 0.001 \\
\hline Carcinoma, diffuse type & $46.65 \%$ & & & 1.14 & $1.01-1.28$ & 0.029 \\
\hline Tubular adenocarcinoma & $60.76 \%$ & & & 1.16 & $0.88-1.54$ & 0.297 \\
\hline Papillary adenocarcinoma, NOS & $75.24 \%$ & & & 0.96 & $0.55-1.66$ & 0.879 \\
\hline Mucinous adenocarcinoma & $51.56 \%$ & & & 0.95 & $0.77-1.18$ & 0.662 \\
\hline Signet ring cell carcinoma & $46.73 \%$ & & & 1.11 & $1.04-1.19$ & 0.003 \\
\hline Stage & & 935.68 & $<0.001$ & & & \\
\hline I & $71.28 \%$ & & & Reference & & \\
\hline II & $54.39 \%$ & & & 1.93 & $1.78-2.11$ & $<0.001$ \\
\hline III & $33.04 \%$ & & & 3.12 & 2.83-3.44 & $<0.001$ \\
\hline Surgery & & 1882.3 & $<0.001$ & & & \\
\hline No surgery & $18.13 \%$ & & & Reference & & \\
\hline Non-Total or Non-near-total gastrectomy & $62.31 \%$ & & & 0.32 & $0.28-0.36$ & $<0.001$ \\
\hline Total or near total gastrectomy & $49.91 \%$ & & & 0.37 & $0.32-0.43$ & $<0.001$ \\
\hline Radiation & & 14.32 & $<0.001$ & & & \\
\hline No radiotherapy & $53.32 \%$ & & & Reference & & \\
\hline Radiotherapy & $53.57 \%$ & & & 0.64 & $0.60-0.69$ & $<0.001$ \\
\hline Radiotherapy unknown & $47.95 \%$ & & & 0.91 & $0.73-1.12$ & 0.357 \\
\hline
\end{tabular}

Abbreviation: NOS= no other specific; SEER=Surveillance, Epidemiology and End Results

aBeing restaged according to the criteria of AJCC Cancer Staging Manual (7th edition, 2010) 


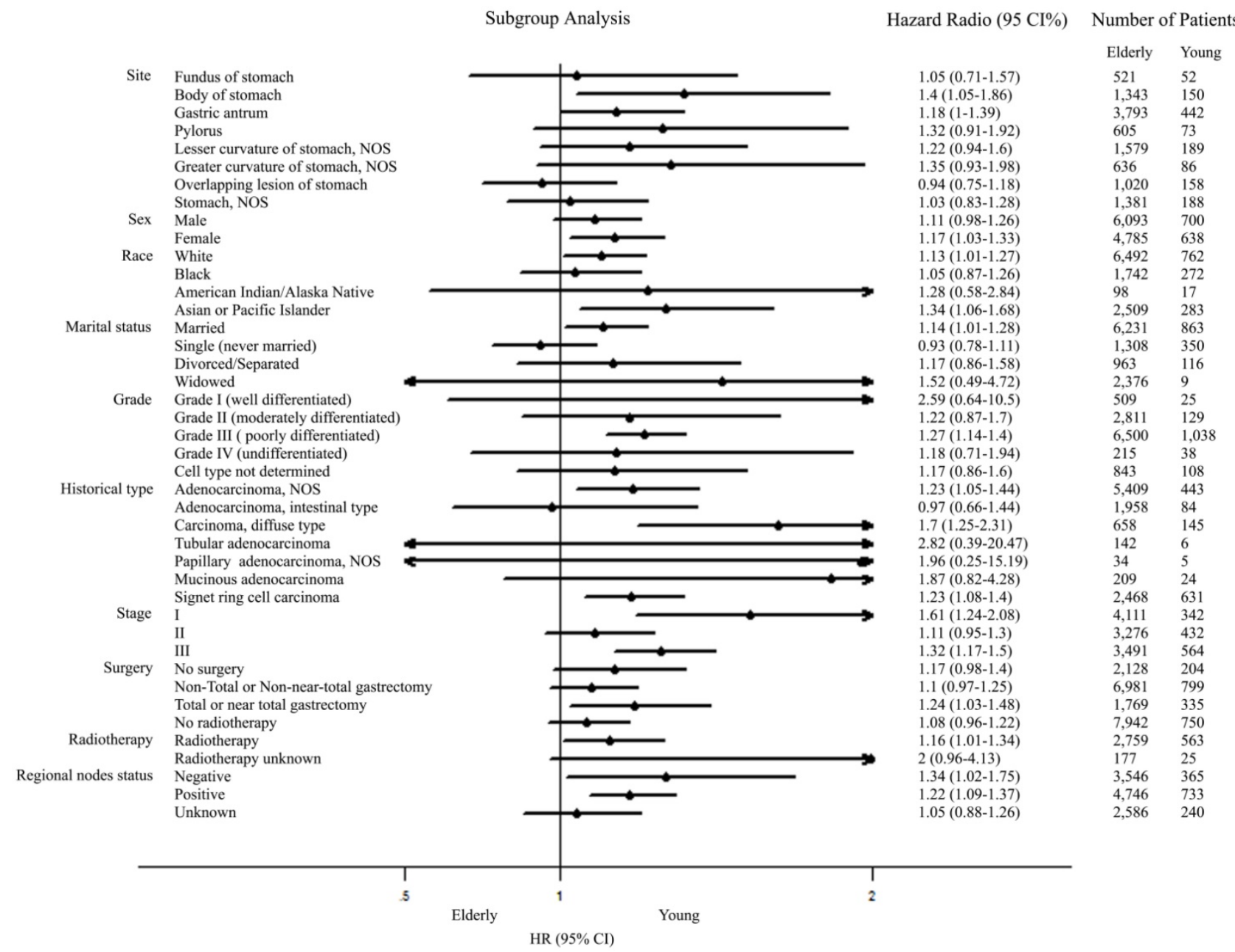

Figure 2: Forest plot summarizing hazard ratios for elderly versus young patients in subgroup analyses. The $\mathrm{X}$-axis displays the hazard ratio and $95 \%$ $\mathrm{Cl}$ of each subgroup, ticks are arranged at $0.1,0.5,1.0$ and 2 .

Kaplan-Meier survival curve in 1:1 matched non-metastatic gastric cancer

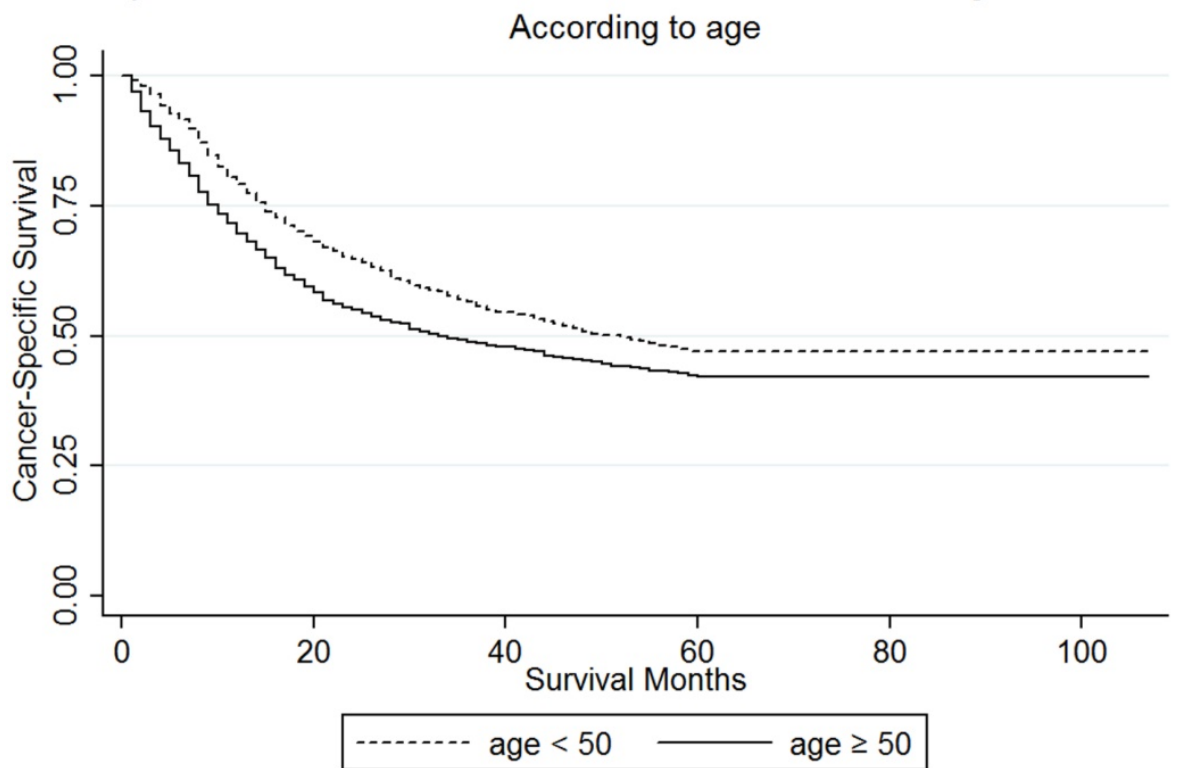

Figure 3: Kaplan-Meier survival curves: The cancer-specific survival of patients with M0 gastric cancer according to age in 1:1 matching, young versus elderly patients. $X 2=17.65, P<0.001$ 
Table 4. Characteristics of patients with non-metastatic gastric cancer by age in 1:1 matching, young versus elderly. SEER 2004-2012 $(n=2676)^{a}$

\begin{tabular}{|c|c|c|c|c|}
\hline \multirow[t]{2}{*}{ Characteristics } & \multirow{2}{*}{$\begin{array}{l}\text { Total } \\
2676(100)\end{array}$} & \multirow{2}{*}{$\begin{array}{l}<50 \\
1338(50)\end{array}$} & \multirow{2}{*}{$\begin{array}{l}\geq 50 \\
1338(50)\end{array}$} & \multirow[t]{2}{*}{ Pvalue } \\
\hline & & & & \\
\hline \multicolumn{5}{|l|}{ Median Follow-up } \\
\hline Months (IQR) & & $21(9-48)$ & $20(7-74)$ & \\
\hline Age (IQR) & 49 (44-73) & $44(39-47)$ & $73(64-80)$ & \\
\hline Sex & & & & 0.938 \\
\hline male & $1402(52.39)$ & $700(52.32)$ & $702(52.47)$ & \\
\hline female & $1274(47.61)$ & $638(47.68)$ & $636(47.53)$ & \\
\hline Race & & & & 0.965 \\
\hline white & $1533(57.29)$ & $762(56.95)$ & $771(57.62)$ & \\
\hline black & $537(20.07)$ & $272(20.33)$ & $265(19.81)$ & \\
\hline $\begin{array}{l}\text { American Indian/Alaska } \\
\text { Native }\end{array}$ & $31(1.16)$ & $17(1.27)$ & $14(1.05)$ & \\
\hline Asian or Pacific Islander & $568(21.23)$ & $283(21.15)$ & $285(21.3)$ & \\
\hline Unknown & $7(0.26)$ & $4(0.3)$ & $3(0.22)$ & \\
\hline Marital status & & & & $<0.001$ \\
\hline Married & $1642(61.36)$ & $863(64.5)$ & 779 (58.22) & \\
\hline Single (never married) & $521(19.47)$ & $350(26.16)$ & $171(12.78)$ & \\
\hline Divorced/Separated & $257(9.6)$ & $116(8.67)$ & $141(10.54)$ & \\
\hline Widowed & $256(9.57)$ & $9(0.67)$ & $247(18.46)$ & \\
\hline Histological type & & & & 0.926 \\
\hline Adenocarcinoma, NOS & $890(33.26)$ & $443(33.11)$ & $447(33.41)$ & \\
\hline $\begin{array}{l}\text { Adenocarcinoma, intestinal } \\
\text { type }\end{array}$ & $174(6.5)$ & $84(6.28)$ & $90(6.73)$ & \\
\hline Carcinoma, diffuse type & $281(10.5)$ & $145(10.84)$ & $136(10.16)$ & \\
\hline Tubular adenocarcinoma & $10(0.37)$ & $6(0.45)$ & $4(0.3)$ & \\
\hline $\begin{array}{l}\text { Papillary adenocarcinoma, } \\
\text { NOS }\end{array}$ & $11(0.41)$ & $5(0.37)$ & $6(0.45)$ & \\
\hline Mucinous adenocarcinoma & $55(2.06)$ & $24(1.79)$ & $31(2.32)$ & \\
\hline Signet ring cell carcinoma & $1255(46.9)$ & $631(47.16)$ & $624(46.64)$ & \\
\hline Site & & & & 0.949 \\
\hline Fundus of stomach & $113(4.22)$ & $52(3.89)$ & $61(4.56)$ & \\
\hline Body of stomach & $292(10.91)$ & $150(11.21)$ & $142(10.61)$ & \\
\hline Gastric antrum & $888(33.18)$ & $442(33.03)$ & $446(33.33)$ & \\
\hline Pylorus & $152(5.68)$ & $73(5.46)$ & $79(5.9)$ & \\
\hline $\begin{array}{l}\text { Lesser curvature of stomach, } \\
\text { NOS }\end{array}$ & $384(14.35)$ & $189(14.13)$ & 195 (14.57) & \\
\hline $\begin{array}{l}\text { Greater curvature of stomach, } \\
\text { NOS }\end{array}$ & $174(6.5)$ & $86(6.43)$ & $88(6.58)$ & \\
\hline Overlapping lesion of stomach & $300(11.21)$ & $158(11.81)$ & $142(10.61)$ & \\
\hline Stomach, NOS & $373(14)$ & $188(14)$ & $185(14)$ & \\
\hline Stage $^{c}$ & & & & 0.999 \\
\hline I & $684(25.56)$ & $342(25.56)$ & $342(25.56)$ & \\
\hline II & $863(32.25)$ & $432(32.29)$ & $431(32.21)$ & \\
\hline III & $1129(42.19)$ & $564(42.15)$ & $565(42.23)$ & \\
\hline Grade & & & & 0.943 \\
\hline Grade I & $48(1.79)$ & $25(1.87)$ & $23(1.72)$ & \\
\hline Grade II & $267(9.98)$ & $129(9.64)$ & $138(10.31)$ & \\
\hline Grade III & $2072(77.43)$ & $1038(77.58)$ & $1034(77.28)$ & \\
\hline Grade IV & $71(2.65)$ & $38(2.84)$ & $33(2.47)$ & \\
\hline Cell type not determined & $218(8.15)$ & $108(8.07)$ & $110(8.22)$ & \\
\hline Surgery & & & & 0.004 \\
\hline No surgery & $403(15.06)$ & $204(15.25)$ & 199 (14.87) & \\
\hline $\begin{array}{l}\text { Non-Total or Non-near-total } \\
\text { gastrectomy }\end{array}$ & $1672(62.48)$ & 799 (59.72) & $873(65.25)$ & \\
\hline Total or near total gastrectomy & $601(22.46)$ & $335(25.04)$ & $266(19.88)$ & \\
\hline Radiotherapy & & & & $<0.001$ \\
\hline No radiotherapy & $1612(60.24)$ & $750(56.05)$ & $862(64.42)$ & \\
\hline Radiotherapy & $1015(37.93)$ & $563(42.08)$ & $452(33.78)$ & \\
\hline Radiotherapy unknown & $49(1.83)$ & $25(1.87)$ & $24(1.79)$ & \\
\hline \multicolumn{5}{|c|}{ Abbreviation: NOS= no other specific; SEER=Surveillance, Epidemiology and End } \\
\hline \multicolumn{5}{|c|}{$\begin{array}{l}\text { Results; } Q Q R=1 \text { terquartile range } \\
\text { aData are presented as No. (Percentage) of patients. }\end{array}$} \\
\hline \multicolumn{5}{|c|}{$\begin{array}{l}\text { bP value of the Chi-square test or Wilcoxon-Mann-Whitney test comparing young } \\
\text { and elderly groups }\end{array}$} \\
\hline $\begin{array}{l}\text { 'Being restaged according to the } \\
\text { edition, 2010) }\end{array}$ & e criteria of $\mathrm{AJ}$ & CC Cancer St & aging Manual & \\
\hline
\end{tabular}

Table 5. Univariate and multivariate survival analysis of overall survival (OS) predictors. Chinese cohort 2000-2012 $(n=1037)$

\begin{tabular}{|c|c|c|c|c|c|c|}
\hline \multirow[t]{2}{*}{ Variable } & \multirow[b]{2}{*}{$\begin{array}{l}\text { 3-year } \\
\text { OS }\end{array}$} & \multicolumn{2}{|c|}{$\begin{array}{l}\text { Univariate } \\
\text { Analysis }\end{array}$} & \multicolumn{3}{|c|}{ Multivariate Analysis } \\
\hline & & $\begin{array}{l}\log \\
\operatorname{rank} \chi^{2}\end{array}$ & $\begin{array}{l}\mathbf{P} \\
\text { Value }\end{array}$ & HR & $95 \% \mathrm{CI}$ & $\begin{array}{l}\mathrm{P} \\
\text { Value }\end{array}$ \\
\hline Age & & 19.33 & $<0.001$ & & & \\
\hline$<50$ & $71.44 \%$ & & & \multicolumn{3}{|c|}{ Reference } \\
\hline$\geq 50$ & $52.90 \%$ & & & 1.77 & $1.38-2.28$ & $<0.001$ \\
\hline Sex & & 0.53 & 0.4661 & & & \\
\hline Male & $61.51 \%$ & & & & & \\
\hline Female & $59.25 \%$ & & & & & \\
\hline Lauren classification & & 25.39 & $<0.001$ & & & \\
\hline Intestinal & $63.47 \%$ & & & \multicolumn{3}{|c|}{ Reference } \\
\hline Mixed & $82.05 \%$ & & & 0.46 & $0.23-0.92$ & 0.029 \\
\hline Diffuse & $47.98 \%$ & & & 1.33 & $1.05-1.69$ & 0.018 \\
\hline Depth of invasion & & 218.08 & $<0.001$ & & & \\
\hline $\mathrm{T} 1$ & $95.66 \%$ & & & \multicolumn{3}{|c|}{ Reference } \\
\hline $\mathrm{T} 2$ & $86.82 \%$ & & & 2.07 & $1.1-3.87$ & 0.023 \\
\hline T3 & $47.70 \%$ & & & 4.95 & $2.84-8.64$ & $<0.001$ \\
\hline $\mathrm{T} 4$ & $36.16 \%$ & & & 6.38 & $3.55-11.47$ & $<0.001$ \\
\hline \multicolumn{2}{|c|}{ Lymph node involvement } & 336.7 & $<0.001$ & & & \\
\hline No & $86.91 \%$ & & & \multicolumn{3}{|c|}{ Reference } \\
\hline N1 & $72.95 \%$ & & & 1.62 & $1.13-2.32$ & 0.008 \\
\hline N2 & $47.23 \%$ & & & 3.14 & $2.29-4.3$ & $<0.001$ \\
\hline N3 & $26.32 \%$ & & & 4.61 & $3.42-6.22$ & $<0.001$ \\
\hline Site & & 37.58 & $<0.001$ & & & \\
\hline Upper & $52.89 \%$ & & & \multicolumn{3}{|c|}{ Reference } \\
\hline Middle & $62.10 \%$ & & & 0.78 & $0.6-1.02$ & 0.071 \\
\hline Lower & $65.75 \%$ & & & 0.75 & $0.58-0.97$ & 0.026 \\
\hline Diffuse & $34.01 \%$ & & & 1.27 & $0.86-1.86$ & 0.233 \\
\hline Grade & & 19.25 & $<0.001$ & & & \\
\hline $\begin{array}{l}\text { Undifferentiated/po } \\
\text { or }\end{array}$ & $55.85 \%$ & & & \multicolumn{3}{|c|}{ Reference } \\
\hline Well/moderate & $69.33 \%$ & & & 0.91 & $0.72-1.15$ & 0.434 \\
\hline Unknown & $67.68 \%$ & & & 0.60 & $0.38-0.95$ & 0.027 \\
\hline
\end{tabular}

A great number of studies have focused on the influence of age on cancer. Anders et al. reported that young patients with castrate-resistant prostate cancer showed a worse survival rate despite having fewer comorbidities and a better performance status [25]. Colorectal cancer arising in young patients was reported by Varma et al. to be correlated with an unfavorable prognosis and a higher frequency of mucin-producing tumors and advancement to terminal stages [26]. Cavanaugh-Hussey et al. observed that a younger age was associated with a higher incidence of sentinel lymph node metastasis but a higher survival advantage in melanoma patients [27]. Genetically, it was reported that age at breast cancer diagnosis adds a layer of genomic complexity beyond pathological and clinical variables, including the pattern of somatic mutations, chromosomal copy number variations (CNVs) and transcriptomic profiles [28].

In regard to gastric cancer, most studies reported that young patients presented poorer pathologic behavior and a worse survival rate than elderly patients. Tavares et al. reported that the 5-year survival rate of younger patients with GC was significantly less than elderly patients, along with 
higher proportions of poor differentiation and advanced stage found in the young group [7]. Isobe et al. had reported similar conclusions as Tavares et al $[11,29,30]$. On the contrary, some other research drew different conclusions. Eguchi et al. showed that younger patients with GC had a better, or at least not worse prognosis than elderly patients [9, 15, 31, 32]. However, the statistical significance of most studies was limited by a small size of samples and unbalanced groups. Moreover, considering the difference of incidence and outcome, the analysis based on data of U.S. may not be applicable in Asia. In a multicentric retrospective analysis, we compared the clinicopathological characteristic and survival between young and elderly patients, as well as by using the propensity scoring method to match younger and older patients. The prognostic value of age was validated in patients from the U.S. and China.

In the U.S. cohort, we found that the young GC patients presented unique characteristics, which included more proportion within the group of female and black than the elderly patients, with the addition of poorer histological behavior, including diffuse carcinoma, deep invasion, lymph node involvement, distant metastasis, poor differentiation and advanced stage. Elderly GC patients presented better survival in the stage I IV GC cohort, which probably was associated with their favorable histological phenotypes. Results from this study showed that young age acted as a worse unadjusted prognostic factor of CSS in the stage I IV GC cohort, but a better one in M0 GC. After adjustment, young patients with M0 GC still had an independently better prognosis, especially for those who underwent total gastrectomy. These unique characteristics and survival had a variety of potential underlying etiologies. Neglect of screening in the young population contributes to their advanced stage at diagnosis. This is partly related to the fact that surveillance endoscopy programs were not yet established to identify young GC patients at an earlier stage in US, due to the smaller amounts of youth in GC patients [3]. Young patients with GC were reported to have less frequency of alarm symptoms, which led to a delayed diagnosis as well $[33,34]$. Lower male/female ratio in the young group could be explained by hormonal factors, particularly the harmful role of estrogen [35-39]. More lymph nodes involvement, poorly differentiation and diffuse type were also presented in young patients. A possible molecular mechanism is that GC in young patients is associated with microsatellite instability (MSI) and loss of heterozygosity (LOH) [40, 41]. Moreover, familial gastric cancer is always accompanied by youth oriented tendency and poorer differentiation. Huntsman et al. reported that germ-line truncating mutations in the E-cadherin (CDH1) gene played a role in diffuse type of GC which is more common in younger patients [42]. Although these factors, including delayed stage and poor histologic grade, were defined as independent poor prognostic factors in our multivariate Cox analysis, young patients with M0 GC had a better 3 -year CSS. The opposite role of age on unadjusted CSS revealed that the tendency to metastasis in young GC patients contributed to the poor outcome. To determine underlying mechanisms accounting for a better survival of young patients with M0 GC, we performed subgroup analyses in a forest plot. Age didn't act as a risk factor in some subgroups, which were considerable confounders for age on survival of patients with M0 GC. Among them, surgery was chosen for further research.

Next, we explored if the tendency to undergo surgery contributed towards a better survival in young patients with M0 GC. The percentage of not receiving surgery was $15.25 \%$ and $19.56 \%$ in the young and the elderly group, respectively. However, after the adjusting of surgery and other confounding variables in multivariate Cox analysis, the survival of young patients still showed a significant advantage over elderly patients. We then investigated whether the effect of age on CSS maintained in surgery-stratified analysis. There was no significant difference in CSS between young and elderly patients for those who underwent non-near-total gastrectomies or those who didn't receive any surgeries. Nonetheless, it was found that young age was an independently protective prognostic factor in patients who underwent total gastrectomy by both univariate and multivariate analysis. These findings could be explained by the fact that younger patients with total gastrectomies had better physiological and psychological conditions, which could compensate poorer histology features [15]. Elderly patients are more likely to suffer from many chronic diseases, such as hypertension, diabetes and heart disease, which contribute to their poorer physiological status. Additionally, some elderly patients may be unwilling to receive active treatments. Generally, a fine general status is essential to the chemotherapeutic tolerance and the progression of postoperative recovery. On the contrary, overall condition probably played less of a role in the recovery procession of patients with non-near-total gastrectomies or no surgeries.

In order to reduce a selection bias arising from an imbalance between ages, we carried out a propensity score matching method. After 1:1 matching of young patients with elderly patients by race, sex, histological grade, TNM stage and histological types, we excluded the bias owing to 
inequality between the two groups. In this way, the younger patients have almost the same distributions of selecting covariates as the matching subset of the elderly patients. Younger patients still showed a better outcome than the elderly patients in CSS after the matching up.

Additionally, we would like to determine if the results of our study are limited in the U.S. We enrolled patients from Chinese institutions and performed analysis in M0 GC patients with gastrectomy. Although the proportion of young group was larger than in the U.S. cohort (10.95\% versus $23.53 \%$ ), poorer differentiation and better overall survival of young patients were also observed in Chinese cohort. Due to lack of information, OS, instead of CSS, was treated as the outcome of interest in Chinese cohort. Besides, gastrectomy wasn't divided into non-total and total gastrectomy. However, it indicates our finding, that young patients tend to survive longer especially after surgery despite poorer tumor behavior, can also be applied in China. This is the first retrospective study on a large scale that is focusing on the age-stratified analysis of M0 GC in U.S. and China. Accordingly, our study inevitably has several limitations. Firstly, we didn't adjust some therapeutic confounding factors including chemotherapy or quality of surgery due to the lacking information. Secondly, comorbidities aren't reported in SEER, which may influence the survival and treatment of those affected. Thirdly, Due to lack of data, OS was treated as the outcome of interest instead of CSS while gastrectomy wasn't divided into non-total and total gastrectomy in Chinese cohort.

Notwithstanding the above limitations, our study adds to current knowledge by demonstrating the unique characteristics and survival of younger patients with GC. In U.S. cohort, younger groups showed poorer histological behaviors, advanced terminal stages, higher rates of surgeries and better survival than the elderly in patients without metastasis, in spite of the worse prognosis shown in the survival curve of the stage I IV GC cohort. The different role of age on unadjusted CSS could be explained that the tendencies to metastasis in young GC patients contributed to the worse outcome. Based on data from multiple Chinese centers, it is validated that the conclusion, that young patients with M0 GC tend to survive longer than the elderly after gastrectomy despite poorer tumor behavior, can be also applicable in China. Furthermore, we predicted that the favorable prognosis in younger patients with M0 GC, which was controversial in previous researches, might be a result from a better overall condition acting on progression of postoperative recovery and a tolerance to subsequent chemotherapy. More attention should be paid to advanced screening and early diagnosis in younger patients with GC, since they presented poorer tumor behavior and benefited more from gastrectomies, compared with elderly patients. Considering that unique genetic changes including MSI, LOH and germ-line truncation play a vital part in this phenomenon, it is necessary to instill great importance to take preventive measure of early onset of gastric cancer, including detailed family surveys, genetic testing for high-risk populations, early detection of CDH1 mutations, and preventive gastrectomy for stomachs with canceration tendencies. Developing novel diagnostic biomarkers for gastric cancer is also essential in the future [43].

\section{Abbreviations}

GC: gastric cancer; SEER: Surveillance, Epidemiology and End Results; ICD-O-3: International Classification of Diseases for Oncology, Third Edition; IQR: interquartile range; NOS: no other specific; CSS: cancer-specific survival; OS: overall survival; HR: hazard ratio; CI: confidence interval.

\section{Supplementary Material}

Supplementary tables.

http://www.jcancer.org/v09p1188s1.pdf

\section{Acknowledgements}

The efforts of the SEER Program tumor registries are acknowledged in the creation of the SEER database.

\section{Funding}

This study was supported by the grant from the Hospital Foundation of Fudan University Shanghai Cancer Center (YJ201504).

\section{Ethics approval}

Our study was approved by the Fudan University Shanghai Cancer Center Ethics Committee and Institutional Review Board of Second Military Medical University. Our work was all carried out in compliance with the Helsinki Declaration.

\section{Competing Interests}

The authors have declared that no competing interest exists.

\section{References}

1. Siegel RL, Miller KD, Jemal A. Cancer Statistics, 2017. CA Cancer J Clin. 2017; 67: 7-30.

2. Van Cutsem E, Sagaert X, Topal B, Haustermans K, Prenen H. Gastric cancer. Lancet. 2016; 388: 2654-64.

3. Karimi P, Islami F, Anandasabapathy S, Freedman ND, Kamangar F. Gastric cancer: descriptive epidemiology, risk factors, screening, and prevention. Cancer Epidemiol Biomarkers Prev. 2014; 23: 700-13. 
4. Anderson WF, Camargo MC, Fraumeni JF, Jr., Correa P, Rosenberg PS, Rabkin CS. Age-specific trends in incidence of noncardia gastric cancer in US adults. JAMA. 2010; 303: 1723-8.

5. Parsonnet J, Harris RA, Hack HM, Owens DK. Modelling cost-effectiveness of Helicobacter pylori screening to prevent gastric cancer: a mandate for clinical trials. Lancet. 1996; 348: 150-4.

6. Parsonnet J, Friedman GD, Vandersteen DP, et al. Helicobacter pylori infection and the risk of gastric carcinoma. N Engl J Med. 1991; 325: 1127-31.

7. Tavares A, Gandra A, Viveiros F, Cidade C, Maciel J. Analysis of clinicopathologic characteristics and prognosis of gastric cancer in young and older patients. Pathol Oncol Res. 2013; 19: 111-7.

8. Furukawa H, Iwanaga T, Imaoka S, et al. Multifocal gastric cancer in patients younger than 50 years of age. Eur Surg Res. 1989; 21: 313-8.

9. Kim DY, Ryu SY, Kim YJ, Kim SK. Clinicopathological characteristics of gastric carcinoma in young patients. Langenbecks Arch Surg. 2003; 388: 245-9.

10. Hsieh FJ, Wang YC, Hsu JT, Liu KH, Yeh CN. Clinicopathological features and prognostic factors of gastric cancer patients aged 40 years or younger. J Surg Oncol. 2012; 105: 304-9.

11. Isobe T, Hashimoto K, Kizaki J, et al. Characteristics and prognosis of gastric cancer in young patients. Oncol Rep. 2013; 30: 43-9.

12. Pisanu A, Podda M, Cois A, Uccheddu A. Gastric cancer in the young: is it a different clinical entity? A retrospective cohort study. Gastroenterol Res Pract. 2014; 2014: 125038

13. Kim KH, Kim YM, Kim MC, Jung GJ. Analysis of prognostic factors and outcomes of gastric cancer in younger patients: a case control study using propensity score methods. World J Gastroenterol. 2014; 20: 3369-75.

14. Al-Refaie WB, Hu CY, Pisters PW, Chang GJ. Gastric adenocarcinoma in young patients: a population-based appraisal. Ann Surg Oncol. 2011; 18: 2800-7.

15. Lai JF, Kim S, Li C, et al. Clinicopathologic characteristics and prognosis for young gastric adenocarcinoma patients after curative resection. Ann Surg Oncol. 2008; 15: 1464-9.

16. Lin JT. Screening of gastric cancer: who, when, and how. Clin Gastroenterol Hepatol. 2014; 12: 135-8.

17. Forman D, Burley VJ. Gastric cancer: global pattern of the disease and an overview of environmental risk factors. Best Pract Res Clin Gastroenterol. 2006; 20: 633-49.

18. Ferro A, Peleteiro B, Malvezzi M, et al. Worldwide trends in gastric cancer mortality (1980-2011), with predictions to 2015, and incidence by subtype. Eur J Cancer. 2014; 50: 1330-44

19. Bacani J, Zwingerman R, Di Nicola N, et al. Tumor microsatellite instability in early onset gastric cancer. J Mol Diagn. 2005; 7: 465-77.

20. Kwak HW, Choi IJ, Kim CG, et al. Individual having a parent with early-onset gastric cancer may need screening at younger age. World J Gastroenterol. 2015; 21: 4592-8.

21. Washington K. 7th edition of the AJCC cancer staging manual: stomach. Ann Surg Oncol. 2010; 17: 3077-9.

22. Wang J, Sun Y, Bertagnolli MM. Comparison of gastric cancer survival between Caucasian and Asian patients treated in the United States: results from the Surveillance Epidemiology and End Results (SEER) database. Ann Surg Oncol. 2015; 22: 2965-71.

23. Niu X, Roche LM, Pawlish KS, Henry KA. Cancer survival disparities by health insurance status. Cancer Med. 2013; 2: 403-11.

24. Bertuccio P, Chatenoud L, Levi F, et al. Recent patterns in gastric cancer: a global overview. Int J Cancer. 2009; 125: 666-73.

25. Humphreys MR, Fernandes KA, Sridhar SS. Impact of Age at Diagnosis on Outcomes in Men with Castrate-Resistant Prostate Cancer (CRPC). J Cancer. 2013; 4: 304-14.

26. Varma JR, Sample L. Colorectal cancer in patients aged less than 40 years. J Am Board Fam Pract. 1990; 3: 54-9.

27. Cavanaugh-Hussey MW, Mu EW, Kang S, Balch CM, Wang T. Older Age is Associated with a Higher Incidence of Melanoma Death but a Lower Incidence of Sentinel Lymph Node Metastasis in the SEER Databases (2003-2011). Ann Surg Oncol. 2015; 22: 2120-6.

28. Azim HA, Jr., Nguyen B, Brohee S, Zoppoli G, Sotiriou C. Genomic aberrations in young and elderly breast cancer patients. BMC Med. 2015; 13: 266.

29. Simsa J, Leffler J, Hoch J, Linke Z, Padr R. Gastric cancer in young patients--is there any hope for them? Acta Chir Belg. 2004; 104: 673-6.

30. Theuer CP, de Virgilio C, Keese G, et al. Gastric adenocarcinoma in patients 40 years of age or younger. Am J Surg. 1996; 172: 473-6; discussion 6-7.

31. Eguchi T, Takahashi Y, Yamagata M, Kasahara M, Fujii M. Gastric cancer in young patients. J Am Coll Surg. 1999; 188: 22-6.

32. Kim JH, Boo YJ, Park JM, et al. Incidence and long-term outcome of young patients with gastric carcinoma according to sex: does hormonal status affect prognosis? Arch Surg. 2008; 143: 1062-7; discussion 7.

33. Maconi G, Kurihara H, Panizzo V, et al. Gastric cancer in young patients with no alarm symptoms: focus on delay in diagnosis, stage of neoplasm and survival. Scand J Gastroenterol. 2003; 38: 1249-55.

34. Boldys H, Marek TA, Wanczura P, Matusik P, Nowak A. Even young patients with no alarm symptoms should undergo endoscopy for earlier diagnosis of gastric cancer. Endoscopy. 2003; 35: 61-7.

35. Kojima O, Takahashi T, Kawakami S, Uehara Y, Matsui M. Localization of estrogen receptors in gastric cancer using immunohistochemical staining of monoclonal antibody. Cancer. 1991; 67: 2401-6.
36. Derakhshan $\mathrm{MH}$, Liptrot $\mathrm{S}$, Paul J, Brown IL, Morrison $\mathrm{D}$, McColl KE Oesophageal and gastric intestinal-type adenocarcinomas show the same male predominance due to a 17 year delayed development in females. Gut. 2009; 58 : $16-23$

37. Maeta M, Yamashiro H, Oka A, Tsujitani S, Ikeguchi M, Kaibara N. Gastric cancer in the young, with special reference to 14 pregnancy-associated cases: analysis based on 2,325 consecutive cases of gastric cancer. J Surg Oncol. 1995; 58: $191-5$

38. Chung HW, Noh SH, Lim JB. Analysis of demographic characteristics in 3242 young age gastric cancer patients in Korea. World J Gastroenterol. 2010; 16: 256-63.

39. Lindblad M, Ye W, Rubio C, Lagergren J. Estrogen and risk of gastric cancer: a protective effect in a nationwide cohort study of patients with prostate cancer in Sweden. Cancer Epidemiol Biomarkers Prev. 2004; 13: 2203-7.

40. Shiao YH, Bovo D, Guido M, et al. Microsatellite instability and/or loss of heterozygosity in young gastric cancer patients in Italy. Int J Cancer. 1999; 82: 59-62.

41. Gryfe R, Kim H, Hsieh ET, et al. Tumor microsatellite instability and clinical outcome in young patients with colorectal cancer. N Engl J Med. 2000; 342: 69-77.

42. Huntsman DG, Carneiro F, Lewis FR, et al. Early gastric cancer in young, asymptomatic carriers of germ-line E-cadherin mutations. N Engl J Med. 2001; 344: 1904-9.

43. Wu X, Tan X, Fu SW. May Circulating microRNAs be Gastric Cancer Diagnostic Biomarkers? J Cancer. 2015; 6: 1206-13. 